TecnoLógicas

ISSN-p 0123-7799

ISSN-e 2256-5337

Vol. 21, No. 43, pp. 127-134

Sep-dic de 2018

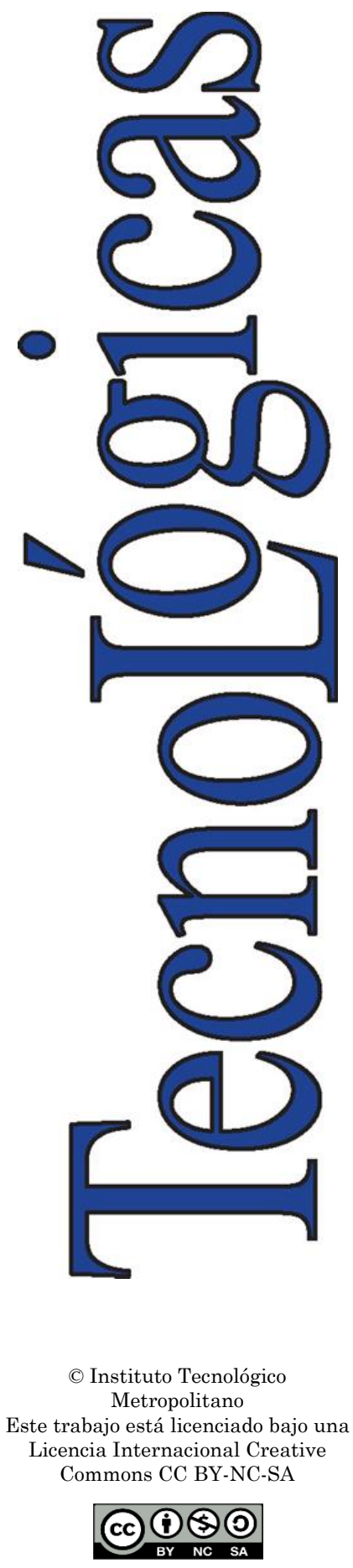

Artículo de Investigación/Research Article

\section{Efecto del tiempo de almacenamiento y sistema de envase en el color del pimentón verde (Capsicum annuum) mínimamente procesado}

\section{Effect of storage time and packaging system on minimally processed green pepper (Capsicum annuum)}

\author{
Natalí López-Mejía ${ }^{1}$ y Luis Eduardo Ordóñez-Santos ${ }^{2}$
}

Recibido: 21 de noviembre de 2017

Aceptado: 16 de junio de 2018

\section{Cómo citar / How to cite}

N. López-Mejía, y L. E. Ordoñez-Santos, Efecto del tiempo de almacenamiento y sistema de envase en el color del pimentón verde (Capsicum annuum) mínimamente procesado. TecnoLógicas, vol. 21, no. 43, pp. 127-134, 2018.

MSc. en Ingeniería Agroindustrial, Ingeniera Agroindustrial, Facultad de Ingeniería y Administración, Departamento de Ingeniería, Universidad Nacional de Colombia, Palmira-Colombia, nalopezme@unal.edu.co

2 PhD. en Ciencias Biológicas, Facultad de Ingeniería y Administración, Departamento de Ingeniería, Universidad Nacional de Colombia, PalmiraColombia, leordonezs@unal.edu.co 


\title{
Resumen
}

El pimentón verde es una Solanaceae cultivada en zonas tropicales y empleada como saborizante y colorante natural. Es considerado un vegetal perecedero, que por lo general es almacenado entre $7,5-8{ }^{\circ} \mathrm{C}$, con el fin de evitar acelerar la transpiración del fruto y daños por frío, lo que a su vez afecta la coloración verde característica. En la actualidad, no se encuentran investigaciones sobre la degradación de pigmentos en el Pimentón Mínimamente Procesado (PMP), por lo tanto el objetivo de este estudio fue evaluar el efecto de dos sistemas de envase y tiempo de almacenamiento sobre las características fisicoquímicas del PMP. Para ello, los frutos fueron almacenados sin envase (control), en polietileno de alta densidad (PEAD) y en polietileno de alta densidad con sellado al vacío $(\mathrm{PEAD}-\mathrm{V})$ en condiciones de refrigeración $\left(9{ }^{\circ} \mathrm{C}\right.$ y $\left.45 \% \mathrm{HR}\right)$. Las variables evaluadas fueron pérdida de peso, $\mathrm{pH}$, acidez titulable, concentración de clorofila total y carotenoides totales, en el día 0,7 y 14 de almacenamiento. Los resultados indican que tanto el sistema de envase como el tiempo de almacenamiento tuvieron efecto significativo sobre las características de calidad evaluadas en PMP. Después de 14 días de almacenamiento los frutos de pimentón verde envasados en PEAD-V presentaron una retención del $62 \%$ de las clorofilas, $75 \%$ de los carotenoides totales y menor cambio de color $(\Delta \mathrm{E}=12)$ respecto al tiempo de almacenamiento 0 . Con base en estos resultados se puede concluir que el envase PEAD-V puede ser una alternativa válida en la conservación del PMP.

\section{Palabras clave}

Capsicum annuum, clorofila total, sistema de empaque, atmósfera modificada, conservación de alimentos.

\begin{abstract}
Green peppers, a Solanaceae cultivated in tropical zones, are used as natural food coloring and flavoring. Considered perishable vegetables, they are usually stored between 7.5 and $8^{\circ} \mathrm{C}$ in order to avoid accelerating fruit transpiration and cold damage (chilling injury), which in turn affect their characteristic green coloration. At present, there are no studies available on the degradation of pigments in Minimally Processed Pepper (MPP). Therefore, the objective of this study was to evaluate the effect of two packaging systems and storage time on the physicochemical characteristics of MPP. For this purpose, the fruits were stored without packaging (control group), in high-density polyethylene (HDPE), and in high-density polyethylene with a vacuum seal (HDPE-V) under refrigeration conditions $\left(9^{\circ}\right.$ $\mathrm{C}$ and $45 \% \mathrm{RH})$. The variables weight loss, $\mathrm{pH}$, titratable acidity, total chlorophyll concentration, and total carotenoids were evaluated on days 0,7 and 14 of storage. The results indicate that both packaging system and storage time had a significant effect on the quality characteristics of MPP. After 14 days of storage, the green pepper fruits packed in HDPE-V presented a retention of $62 \%$ of the chlorophylls, $75 \%$ of the total carotenoids, and less color change $(\Delta \mathrm{E}=12)$ with respect to time to storage time 0 . Based on these results, it can be concluded that the HDPE-V container can be a suitable alternative to preserve MPP.
\end{abstract}

\section{Keywords}

Capsicum annuum, total chlorophyll, packaging system, modified atmosphere, food preservation. 


\section{INTRODUCCIÓN}

El pimentón (Capsicum annuum) es un vegetal perteneciente a la familia Solanaceae [1], cultivado en zonas tropicales y subtropicales del mundo. Este es consumido en estado fresco $y$ es empleado como saborizante y colorante natural [2]. Es considerado fuente importante de fibra, vitaminas A y C y fenoles, lo que le confiere propiedades antioxidantes y anti-inflamatorias [3]. Los frutos presentan diferentes colores según su estado de madurez, desde color verde a amarillo y rojo cuando está maduro.

La coloración verde en los frutos de pimentón es atribuida principalmente a la presencia de clorofila $a \mathrm{y}$ b en una proporción de 3:1, la cual se encuentra en el cloroplasto y también a propiedades físicas, tales como la disposición de las células en la superficie que selectivamente absorben y reflejan la luz incidente visible produciendo un espectro característico [4]. Este atributo de color es una de las características más importantes para el consumidor, ya que permite determinar la aceptación y la frescura del pimentón verde. Sin embargo, durante el procesamiento y almacenamiento de los productos mínimamente procesados, las operaciones físicas de pelado y cortado aceleran los daños fisiológicos en los frutos. Estas operaciones desencadenan cambios de color en el producto al degradar los pigmentos y generar compuestos pardos. Alteraciones causadas principalmente por el ingreso de oxígeno en los tejidos, y la acción de las enzimas [5].

El efecto del almacenamiento sobre las características fisicoquímicas del pimentón mínimamente procesado ha sido abordado en diferentes estudios, Barbagallo, Crisari y Patamé [6] comentan que el pimentón verde frente a los frutos rojos y amarillos, presentó sensorialmente mayor grado de aceptación después de 30 días de almacenamiento. En otro estudio realizado por Kugle et al. [7], reportan una reducción del $30 \%$ en la concentración de carotenoides en pimentón amarillo mínimamente procesado después de 14 días de almacenamiento a $5{ }^{\circ} \mathrm{C}$ y $90 \% \mathrm{HR}$. Adicionalmente, Ranjitha, Rao, Shivashankara y Roy [8] reportan que los parámetros de color $L^{*} a^{*} y b^{*}$ en pimentón verde mínimamente procesado empacado en atmosfera modificada, no presentó diferencias significativas frente a los frutos frescos después de 9 días de almacenamiento a $8^{\circ} \mathrm{C}$.

Existen muy pocos trabajos de investigación sobre los cambios de color en pimentón verde mínimamente procesado, producto con alta demanda en el mercado gourmet, los cuales están interesados en ofrecer a sus consumidores alimentos frescos y de alta calidad. Por lo tanto, el objetivo de la presente investigación fue evaluar el efecto del sistema de envasado y el tiempo de almacenamiento en refrigeración, sobre el color del pimentón verde mínimamente procesado.

\section{MATERIALES Y MÉTODOS}

\subsection{Materia prima}

Pimentón verde (Capsicum annuum) proveniente de un mismo lote, de tamaño uniforme, sin defectos ni enfermedades se adquirió de un mercado local (CAVASA, Candelaria, Colombia) y procesado en el laboratorio de frutas y hortalizas de la Universidad Nacional de Colombia Sede Palmira. Estos se lavaron y desinfectaron a $50 \mathrm{ppm}$ con hipoclorito de sodio por inmersión durante $10 \mathrm{~min}$. El fruto se sometió a tratamiento térmico por $1 \mathrm{~min}$ a $70{ }^{\circ} \mathrm{C}$ para disminuir carga microbiana [9]. Posteriormente, se acondicionaron en tamaños homogéneos, en forma de julianas con $15 \mathrm{~mm}$ de largo y $10 \mathrm{~mm}$ de ancho empleando una procesadora de vegetales (Javar, Colombia). Luego se dejaron secar a condiciones ambientales $\left(28^{\circ} \mathrm{C}\right.$ y $65 \% \mathrm{HR}$ por $30 \mathrm{~min}$ ), con el fin de eliminar el exceso 
de humedad superficial antes de empacar. Las muestras fueron almacenadas empleando diferentes sistemas de envase, bajo condiciones de refrigeración $\left(9^{\circ} \mathrm{C}\right.$ y 45 \%HR por 14 días).

\subsection{Envase y almacenamiento}

Dos sistemas de envase fueron evaluados en el Pimentón verde Mínimamente Procesado (PMP). Para el sistema 1 (empacado en material de polietileno de alta densidad - envase en PEAD sin vacío), las muestras fueron incorporadas manualmente dentro del envase (capacidad $=100 \mathrm{~g}$ ) y este fue sellado mediante el sistema ziploc de las mismas. Para el sistema 2 (envase en PEAD bajo vacío) las muestras fueron incorporadas de igual forma y selladas bajo vacío empleando un equipo (EGARVAC S.C.P, Basic B, España) con bomba de vacío de $20 \mathrm{~m}^{3} / \mathrm{h}$. Finalmente, para el sistema control (sin envase - control), las muestras fueron incorporadas en una bandeja de poliestireno comercial sin ninguna barrera a las condiciones de almacenamiento. Para todos los tratamientos se empleó $61 \pm 1,50 \mathrm{~g}$ de muestra. Propiedades del material de envase como Permeabilidad al Vapor de Agua (PVA) y al Oxígeno (PO) fueron tomadas de lo reportado por Polymer Properties Data base [10] $(\mathrm{PVA}=5,9$ $\mathrm{g} 25 \mu / \mathrm{m}^{2} / 24 \mathrm{~h} \quad$ a $25 \quad{ }^{\circ} \mathrm{C} ; \quad \mathrm{PO}_{2}=2325$ $\left.\mathrm{g} 25 \mu / \mathrm{m}^{2} / 24 \mathrm{~h}\right)$.

\subsection{Análisis fisicoquímicos}

El peso del PMP fue medido empleando una balanza analítica de precisión $\pm 0,001$ g (Boeco, BBL31, Alemania). Para ello previamente el material vegetal fue extraído del envase y climatizado $\left(28^{\circ} \mathrm{C}\right)$. Parámetros como $\mathrm{pH}$ y acidez titulable (\% de ácido cítrico) se cuantificaron siguiendo los métodos AOAC 981.12 y 942.15 [11] respectivamente.
La medición de la concentración de clorofila total (CloT) expresada en $\mathrm{mg} / 100$ g de PMP se realizó de acuerdo con el método reportado por Gokmen, Bahçeci y Acar [12], con algunas modificaciones. Para ello, se utilizaron muestras de PMP de $5 \mathrm{~g}$, las cuales se incorporaron a tubos falcón de $25 \mathrm{~mL}$ y se mezclaron con $20 \mathrm{~mL}$ de acetona al $80 \%(\mathrm{v} / \mathrm{v})$. Dicha mezcla fue homogenizada a $15.000 \mathrm{rpm}$ por $1 \mathrm{~min}$ empleando ultraturrax (IKA, T18 digital, Alemania) y centrifugada (Indulab, 08T, Colombia) a $8.000 \mathrm{rpm} \mathrm{a} 4{ }^{\circ} \mathrm{C}$ por $5 \mathrm{~min}$. El extracto obtenido fue filtrado usando papel filtro Whatman No.1. La absorbancia fue medida a $645 \mathrm{~nm}\left(\mathrm{~A}_{645}\right)$ y $663 \mathrm{~nm}\left(\mathrm{~A}_{663}\right)$ [13] usando un espectrofotómetro (Jenway, 6320 D, USA). La concentración fue hallada con base en la ley de Lambert - Beer (1).

$$
\operatorname{CloT}\left(\frac{\mathrm{mg}}{100 \mathrm{~g}}\right)=\frac{\left(20,2 * \mathrm{~A}_{645}+8,02 * \mathrm{~A}_{663}\right) * \mathrm{FD}}{10 * \mathrm{~W}}
$$

La concentración de carotenoides totales (CT) fue medida en el mismo extracto obtenido para la cuantificación de clorofila total. Para ello, se midió la absorbancia a $470 \mathrm{~nm}\left(\mathrm{~A}_{470}\right)$ y se realizó el respectivo cálculo (2) [14].

$$
\mathrm{CT}=\frac{1000 * \mathrm{~A}_{470}-3,27 \text { Cloa }-104 \text { Clob } * \mathrm{FD}}{2290 * \mathrm{~W}}
$$

Donde,

$\mathrm{FD}=$ Factor de dilución

$\mathrm{W}=$ peso de la muestra en gramos

Parámetros de color CIEL*a*b* fueron analizados en PMP por triplicado con un colorímetro triestímulo (Chroma MeterCR-400, Konica Minolta, Japón) previamente calibrado $(\mathrm{Y}=89,5, \mathrm{x}=0,3166 \mathrm{y}$ y=0,3347). Para el análisis se empleó un iluminante D65 y ángulo de observador $2^{\circ}$ [15]. Valores de croma (C), ángulo de tono $\left(\mathrm{h}^{\circ}\right)$ y diferencia de color $(\Delta \mathrm{E})$ fueron determinados mediante las ecuaciones (3), (4) y (5) respectivamente [16]. 
$\mathrm{C}=\sqrt{\mathrm{a} *^{2}+\mathrm{b} *^{2}}$

$\mathrm{h}^{\circ}=\operatorname{arctg} \frac{\mathrm{b}^{*}}{\mathrm{a}^{*}}+180^{\circ}$

$\Delta \mathrm{E}=\sqrt{\left(\mathrm{a}_{0}-\mathrm{a}\right)^{2}+\left(\mathrm{b}_{0}-\mathrm{b}\right)^{2}+\left(\mathrm{L}_{0}-\mathrm{L}\right)^{2}}$

Donde,

$\mathrm{a}^{*}=$ coloración rojiza $(+)$ y verde $(-)$,

$\mathrm{b}^{*}=$ coloración amarilla $(+)$ y azul (-).

$\Delta \mathrm{E}=$ diferencia de color por efecto del tiempo, siendo $\mathrm{L}_{0}{ }^{*}, \mathrm{a}_{0}{ }^{*} \mathrm{y} \mathrm{b}_{0}{ }^{*}$ los parámetros medidos en el día 0 de almacenamiento.

\subsection{Diseño experimental y análisis estadístico}

Un diseño completamente aleatorizado con arreglo factorial $3^{2}$ fue llevado a cabo para estudiar el efecto del factor tiempo (0, 7 y 14 días) y sistema de envase (control, PEAD y PEAD-V) sobre las variables fisicoquímicas, $\mathrm{pH}$, acidez, clorofila total, carotenoides totales y color, del pimentón mínimamente procesado. El método estadístico consistió en un análisis de varianza ANOVA con comparación de Tukey, empleando un nivel de significancia $\mathrm{P}<0,05$. El software utilizado fue SPSS. 18.

\section{RESULTADOS Y DISCUSIÓN}

\subsection{Análisis fisicoquímico}

El PMP en el día 0 de almacenamiento presentó valores de $\mathrm{pH}$ desde 5,09 hasta 5,19 ; acidez titulable en un intervalo de 0,027 hasta 0,030 ; clorofila total entre 11,15 hasta $13,01 \mathrm{mg} / 100 \mathrm{~g}$ y carotenoides totales en un rango de 3,25 hasta 3,71 $\mathrm{mg} / 100 \mathrm{~g}$. Mayores valores de $\mathrm{pH}$, acidez y menores concentraciones de clorofila total han sido reportados por otros investigadores [17] $(\mathrm{pH}=5,14 \pm 0,05 ; \mathrm{A}$. Cítrico $0,028 \pm 0,001 \%$ y CloT $=6,06$ $\mathrm{mg} / 100 \mathrm{~g})$. Los parámetros de color CIEL*a*b* en PMP para las coordenadas $L^{*}(42,30$ a 44,52$), a *(-11,96$ a $-10,17), b^{*}$ $(14,82$ a 23,79$), C(17,98$ a 28,52$)$ y $h^{\circ}$ $(123,49$ a 126,91), fueron diferentes a los obtenidos por Quipo-Muñoz et al. [17] $\left(\mathrm{L}^{*}=\right.$ $32,32, \mathrm{a}^{*}=-9,91, \mathrm{~b}^{*}=10,20, \mathrm{C}=14,22 \mathrm{y} \mathrm{h}^{\circ}$ $=134,20)$. Las diferencias en las propiedades fisicoquímicas evaluadas pueden ser ocasionadas por el tratamiento térmico al PMP, el cual fue de $70{ }^{\circ} \mathrm{C}$ durante un 1 min para este estudio, menor en comparación al tratamiento térmico de $98^{\circ} \mathrm{C}$ durante 5 min de los autores citados. $\mathrm{El}$ tratamiento térmico al cual fue sometido PMP de este estudio conservó en mayor proporción la concentración de clorofila, ya que esta fue mayor $(11,15$ $13,01 \mathrm{mg} / 100 \mathrm{~g})$ a la reportada en el trabajo de [17] $(6,06 \mathrm{mg} / 100 \mathrm{~g})$. Este comportamiento ya ha sido reportado previamente por autores como MartínezHernández, Artés-Hernández, Gómez, y Artés [18], los cuales argumentan que mayores valores del parámetro a* (más negativo) se debe a mayor concentración del pigmento (clorofila), lo que indica menor pérdida de magnesio y por lo tanto menor formación de feofitinas [19]. Por otro lado, dichas diferencias pueden deberse a la variedad utilizada por el autor y el tiempo de cosecha que llevaba el mismo antes del procesamiento.

Propiedades fisicoquímicas medidas en el pimentón verde mínimamente procesado en función del sistema de envase y del tiempo de almacenamiento se muestran en la Fig. 1, los cuales registraron un efecto significativo $\quad(\mathrm{P}<0,05) \quad$ sobre dichas propiedades. En la Fig. 1a, se muestra cómo la muestra control presentó una disminución significativa de peso en el día 7 de almacenamiento, el cual fue del $89 \%$ aproximadamente. A partir de ese día, PMP solo disminuyó un $17 \%$ más de su peso inicial. Por el contrario, los demás sistemas de envase (PEAD y PEAD-V), no presentaron una disminución significativa en este parámetro (Fig. 1a). La pérdida de 
peso en las muestras control, se debe migración de vapor de agua de la muestra estudio al aire circundante, gracias al gradiente de humedad entre la muestra y un ambiente de almacenamiento con menor humedad (45 \%HR). Por el contrario, en los productos envasados (PEAD y PEAD-V) la baja permeabilidad al vapor de agua del material de envase evito pérdidas de agua.

En la Fig. 1b se muestra cómo el $\mathrm{pH}$ disminuyó significativamente con el tiempo de almacenamiento sobre todo en PMP envasado en el sistema PEAD y PEAD-V, lo que se relaciona con la acidez, la cual aumentó significativamente $(\mathrm{P}<0,05)$ (Fig. 1c). Lo anterior, es debido a la baja permeabilidad al vapor de agua del material de envase, lo que hace que el agua en forma de vapor que va perdiendo el alimento no migre al medio exterior, si no que condense y forme un caldo rico en nutrientes que facilita la proliferación de microorganismos. Adicionalmente, PMP envasado en PEAD-V al no contener oxígeno, y el alimento tener un alto contenido de humedad y de actividad de agua, puede presentar crecimiento de microorganismos que generan procesos de fermentación anaeróbicas. Por lo tanto, en futuros estudios se podría evaluar la estabilidad microbiológica de PMP envasado en los diferentes sistemas de empaque y determinar qué microorganismo está ocasionando la alteración del alimento.
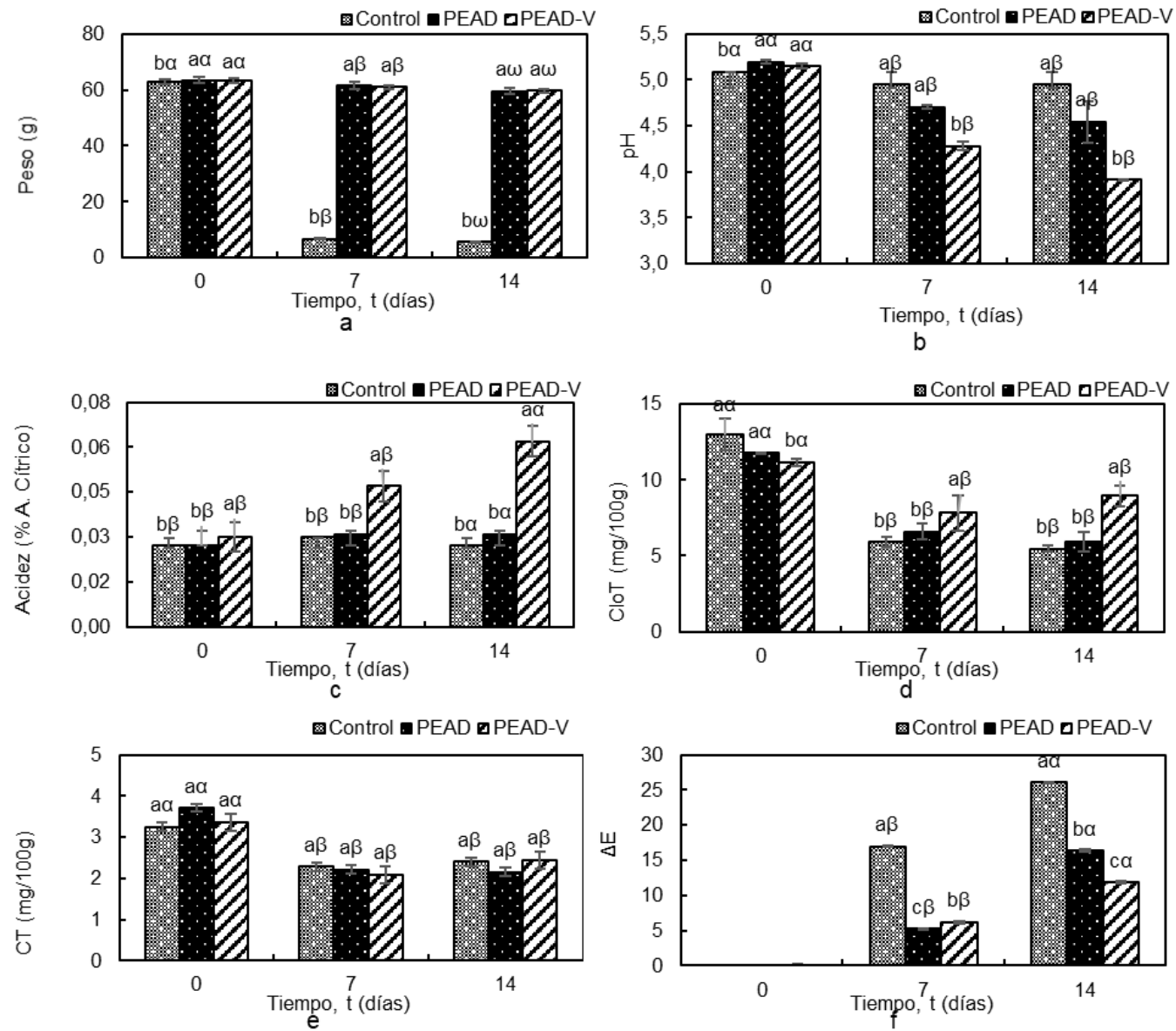

Fig. 1. Propiedades fisicoquímicas del pimentón verde mínimamente procesado en función del sistema de envase y del tiempo de almacenamiento. (a) Peso, (b) pH, (c) acidez titulable (\%A. Cítrico), (d) clorofila total, (e) carotenoides totales y (f) diferencia de color. Letras diferentes en columnas con diferente trama indican diferencias significativas por efecto del sistema de envase. Letras diferentes en columnas con la misma trama incidan diferencias significativas por efecto del tiempo de almacenamiento.

Fuente: autores. 
En la Fig.1d se observa que la clorofila se degradó en mayor proporción en PMP sin envase (control) y en menor proporción en PEAD-V, a pesar de que estas presentaron un menor valor de $\mathrm{pH}$, siendo la clorofila más estable a pH alcalino [20]. En cuanto a la concentración de carotenoides totales, esta solo fue afectada significativamente por el tiempo de almacenamiento (Fig. 1e). Adicionalmente, $\Delta \mathrm{E}$ fue mayor para PMP control respecto a los otros dos sistemas de envase (Fig. 1f). Menor valor en dicho parámetro fue obtenido para PEAD-V en el día 14 de almacenamiento. Los cambios de color en el producto mínimamente procesado se deben principalmente a degradaciones oxidativas de los pigmentos presentes en el pimentón verde, causadas principalmente por las enzimas clorofilasa, lipoxigenasa y peroxidasa [7]. En el caso de las clorofilas, estas pueden ser degradadas enzimáticamente a feofitina seguida de feorfórbido que finalmente se convierten en compuestos incoloros, y son transportados y almacenados en la vacuola [21] y [22]. Respecto a los carotenoides, estos pigmentos son sensibles a procesos de oxidación desencadenados por la lipoxigenasa, presencia de luz, oxígeno y bajos valores de $\mathrm{pH}$ [23]. Las acciones de estos factores oxidan los carotenoides a compuestos incoloros como epoxicarotenoides, apo-carotenoides, e hidroxicarotenoides [24].

\section{CONCLUSIONES}

Después de 14 días de almacenamiento el envase PEAD-V permitió retener el $62 \%$ de las clorofilas, el $75 \%$ de los carotenoides totales y presentó el menor cambio de color $(\Delta \mathrm{E}=12)$ en el pimentón mínimamente procesado. El envase PEAD-V puede ser una alternativa válida en la conservación del pimentón verde mínimamente procesado ya que logró disminuir en gran medida la pérdida de peso del vegetal, así como la degradación de pigmentos como clorofila y carotenoides totales.

\section{AGRADECIMIENTOS}

Este trabajo fue financiado por la Universidad Nacional de Colombia Sede Palmira y desarrollado en el laboratorio de frutas y hortalizas.

\section{REFERENCIAS}

[1] X. Meng, M. Zhang, and B. Adhikari, "Extending shelf-life of fresh-cut green peppers using pressurized argon treatment," Postharvest Biol. Technol., vol. 71, pp. 13-20, 2012.

[2] R. Arimboor, R. B. Natarajan, K. R. Menon, L. P. Chandrasekhar, and V. Moorkoth, "Red pepper (Capsicum annuum) carotenoids as a source of natural food colors: analysis and stability a review," J. Food Sci. Technol., vol. 52, no. 3, pp. 1258-1271, 2015.

[3] S. Chatterjee, Z. Niaz, S. Gautam, S. Adhikari, P. S. Variyar, and A. Sharma, "Antioxidant activity of some phenolic constituents from green pepper (Piper nigrum L.) and fresh nutmeg mace (Myristica fragrans)," Food Chem., vol. 101, no. 2, pp. 515-523, 2007.

[4] J. E. Lancaster, C. E. E. Lister, P. F. Reay, and C. M. Triggs, "Influence of Pigment Composition On Skin Color in a Wide Range of Fruit and Vegetables," Journal of American Society Horticultural science, vol. 4, no. 122. pp. 594-598, 1997.

[5] M. Robles-sánchez, S. Gorinstein, O. Martínbelloso, H. Astiazarán-garcía, and G. G. Reinaldo, "Interciencia," pp. 1-10, 2018.

[6] R. N. Barbagallo, M. Chisari, and C. Patané, "Polyphenol oxidase, total phenolics and ascorbic acid changes during storage of minimally processed 'California Wonder' and 'Quadrato d'Asti' sweet peppers,' LWT - Food Sci. Technol., vol. 49, no. 2, pp. 192-196, 2012.

[7] R. A. Kluge et al., "Qualidade de pimentões amarelos minimamente processados tratados com antioxidantes," Semin. Agrar., vol. 35, no. 2, pp. 801-811, 2014.

[8] K. Ranjitha, D. V Sudhakar Rao, K. S. Shivashankara, and T. K. Roy, "Effect of pretreatments and modified atmosphere packaging on the shelf life and quality of fresh- cut green bell pepper," J. Food Sci. 
Efecto del tiempo de almacenamiento y sistema de envase en el color del pimentón verde (Capsicum annuum) mínimamente procesado

Technol., vol. 52, no. 12, pp. 7872-7882, 2015.

[9] M. Hernández-Carrión, I. Hernando, I. Sotelo-Díaz, M. X. Quintanilla-Carvajal, and a. Quiles, "Use of image analysis to evaluate the effect of high hydrostatic pressure and pasteurization as preservation treatments on the microstructure of red sweet pepper," Innov. Food Sci. Emerg. Technol., vol. 27, pp. 69-78, 2015.

[10] Polymer Properties Database, "Barrer properties of polymers," Polymer Properties Database, 2015. .

[11] W. Horwitz, Official Methods of Analysis of the Association of Official Agricultural Chemists. Soil Sciense, 1956.

[12] V. Gokmen, S. Bahçeci, and J. Acar, "Liquid Chromatographic Method for the Determination of Chlorophylls, Carotenoids, and Their Derivatives in Fresh and Processed Vegetables," J. Liq. Chromatogr. Relat. Technol., vol. 25, no. 8, pp. 1201-1213, 2002.

[13] J. Bruuinsma, "The quantitative analysis of chlorophylls a and b in plant extracts," Photochem. Photobiol., vol. 2, no. 18, pp. 241-249, 1963.

[14] H. Lichtenthaler and A. Wellburn, "Determinations of total carotenoids and chlorophylls b of leaf extracts in different solvents," Biochem. Soc. Trans., vol. 11, no. 1955, pp. 591-592, 1983.

[15] L. E. Ordóñez-Santos, J. Martínez-Girón, and M. E. Arias-Jaramillo, "Effect of ultrasound treatment on visual color, vitamin C, total phenols, and carotenoids content in Cape gooseberry juice," Food Chem., vol. 233, pp. 96-100, 2017.

[16] F. Mendoza, P. Dejmek, and J. M. Aguilera, "Calibrated color measurements of agricultural foods using image analysis," Postharvest Biol. Technol., vol. 41, no. 3, pp. 285-295, 2006.
[17] F. Quipo Muñoz, Á. Ramirez Muñoz, J. Rojas Perez, and L. Ordóñez Santos, "Cambios en la Vitamina $\mathrm{C}$ y el Color durante la Cocción del Pimentón Verde ( Capsicum Annuum L ) Changes in," TecnoLógicas, vol. 31, no. 31, pp. 141-150, 2013.

[18] G. B. Martínez-Hernández, F. ArtésHernández, P. A. Gómez, and F. Artés, "Induced changes in bioactive compounds of kailan-hybrid broccoli after innovative processing and storage," J. Funct. Foods, vol. 5, no. 1, pp. 133-143, 2013.

[19] N. Turkmen, E. S. Poyrazoglu, F. Sari, and Y. Sedat Velioglu, "Effects of cooking methods on chlorophylls, pheophytins and colour of selected green vegetables," Int. J. Food Sci. Technol., vol. 41, no. 3, pp. 281288, 2006.

[20] A. C. Company, "Protection of natural green pigment in the canning of peas," no. 9, 1813.

[21] B. López-Ayerra, M. Antonia Murcia, and F. Garcia-Carmona, "Lipid peroxidation and chlorophyll levels in spinach during refrigerated storage and after industrial processing," Food Chem., vol. 61, no. 1-2, pp. 113-118, 1998.

[22] P. M. A. Toivonen and D. A. Brummell, "Biochemical bases of appearance and texture changes in fresh-cut fruit and vegetables," Postharvest Biol. Technol., vol. 48, no. 1, pp. 1-14, 2008.

[23] I. Odriozola-Serrano, R. Soliva-Fortuny, and O. Mart??n-Belloso, "Changes of healthrelated compounds throughout cold storage of tomato juice stabilized by thermal or high intensity pulsed electric field treatments," Innov. Food Sci. Emerg. Technol., vol. 9, no. 3, pp. 272-279, 2008.

[24] D. B. Rodriguez-Amaya, "Biosynthesis and metabolism," in Food Carotenoids, Chichester, UK: John Wiley \& Sons, Ltd, 2015, pp. 24-46. 